\title{
DISPONIBILIDADE DE POTÁSSIO PARA AS PLANTAS EM SOLOS DO SUL DO BRASIL ESTIMADA POR MÉTODOS MULTIELEMENTARES ${ }^{(1)}$
}

\author{
Leandro Bortolon $^{(2)}$, Clesio Gianello ${ }^{(3)}$ \& Jairo André Schlindwein ${ }^{(4)}$
}

\begin{abstract}
RESUMO
A avaliação da disponibilidade de $\mathrm{K}$ para as plantas é feita pela estimativa de seus teores na forma trocável. Métodos multielementares aumentam a eficiência nos laboratórios de análises de solo, porém a capacidade de troca de cátions (CTC) do solo pode alterar a predição do K disponível. O objetivo deste trabalho foi estimar o K disponível para o milho e para a soja em solos representativos do Estado do RS, pelos métodos Mehlich-1 ( $\left.\mathbf{M}_{1}\right)$, Mehlich-3 $\left(\mathbf{M}_{3}\right)$, resina de troca iônica (resina) e Acetato de Amônio pH 7,0 ( $\left.\mathrm{AcNH}_{4}\right)$, bem como verificar se a CTC influencia a estimativa da disponibilidade de $\mathrm{K}$ no solo para as plantas. $\mathrm{O}$ experimento foi conduzido em microparcelas a céu aberto e, após 45 dias de cultivo, quantificou-se a matéria seca da parte aérea das plantas e determinou-se o o teor de K no tecido. Foram feitas as correlações entre os teores de K extraídos pelos métodos e a relação com a absorção de $\mathrm{K}$ pelas plantas em todos os solos e separando os solos em classes de CTC. As quantidades de K extraídas pela resina foram aproximadamente

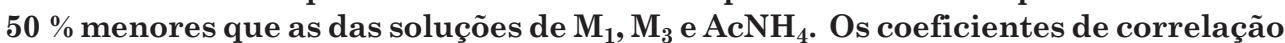
entre os teores de $\mathrm{K}$ extraído pelos métodos tiveram alto grau de associação. A relação entre os teores de $\mathrm{K}$ acumuladas pelas culturas e os teores extraídos pelos métodos não diferiram, independentemente da separação por classes de CTC. A disponibilidade de $\mathrm{K}$ para as plantas em solos do RS pode ser feita pelos métodos testados no presente trabalho. Não houve diferença entre os métodos na avaliação da disponibilidade de $\mathrm{K}$ nas classes de CTC. O método da resina, embora não diferindo dos demais na predição do teor de K disponível, apresenta desvantagem em relação aos demais pela menor capacidade extrativa, o que resulta em classes de disponibilidade menores e propicia o erro analítico.
\end{abstract}

Termos de indexação: métodos de análise de solo, correlação, adubação potássica, soja, milho.

\footnotetext{
(1) Parte da Dissertação de Mestrado do primeiro autor apresentada ao Programa de Pós-Graduação em Ciência do Solo da Universidade Federal do Rio Grande do Sul - UFRGS. Executada com recursos do projeto Laboratório de Análises do Departamento de Solos da Faculdade de Agronomia, UFRGS. Recebido para publicação em maio de 2009 e aprovado em agosto de 2010.

(2) Pós-Doutorando, Universidade do Estado de Santa Catarina - UDESC. Av. Luis de Camões 2090, CEP 88520-000 Lages (SC). Bolsista do CNPq. E-mail: leandro.bortolon@ufrgs.br

(3) Professor Associado do Departamento de Solos, UFRGS. E-mail: cgianello@hotmail.com.br

(4) Professor Adjunto da Universidade Federal de Rondônia - UNIR. Av. Norte Sul 7300, CEP 76940-000 Rolim de Moura (RO). E-mail: jairojas@unir.br
} 


\title{
SUMMARY: AVAILABLE POTASSIUM IN SOILS OF SOUTHERN BRAZIL ESTIMATED BY MULTIELEMENT METHODS
}

\begin{abstract}
Potassium (K) availability to plants is estimated based on the exchangeable K fraction. Multielement methods increase the analysis efficiency in soil laboratories. The cation exchange capacity (CEC) of soils may affect the prediction of available K. Thus, the purpose of this study was to estimate the available K for corn and soybean in soils in the Rio Grande do Sul (RS) state using Mehlich-1 $\left(M_{1}\right)$, Mehlich-3 $\left(M_{3}\right)$, ion-exchange resin (resin), ammonium acetate $\mathrm{pH} 7.0\left(\mathrm{AcNH}_{4}\right)$, and verify whether the CTC affects the estimated availability of soil $\mathrm{K}$ to plants. The experiment was carried out in microplots in the field and after 45 days of growth the shoot dry mass was quantified and K determined in the tissue. The K quantities estimated by the distinct methods were correlated, the relationship with K uptake by plants in all soils established and the soils separated in CTC classes. Resin-extracted K was about $50 \%$ lower than that obtained by $M_{1}, M_{3}$ and $\mathrm{AcNH}_{4}$. There close correlations between the Kquantities extracted by the methods. The relationship between the quantities of $K$ uptake by the crops and the amounts extracted by the tested methods, which did not differ from each other regardless of soil CTC class. Kavailability to plants in soils in RS can be estimated by the methods tested in this study. There was no difference between the evaluation methods of Kavailability in the CTC classes. Despite the resin method being as effective as other methods in estimating available $K$, it has the drawback of a lower extraction capacity, which results in lower values for $K$ availability classes and thus may favor analytical errors.
\end{abstract}

Index terms: soil test methods, correlation, potassium fertilizer, soybean, corn.

\section{INTRODUÇÃO}

A avaliação da disponibilidade de K para as plantas é feita pela estimativa de seus teores na forma trocável, a qual é obtida com a utilização de soluções neutras contendo íons amônio ou sódio, que, por troca iônica, removem a fração considerada trocável. Também são utilizadas soluções ácidas diluídas (Mehlich, 1953, 1984). No entanto, para um método ser eficiente na avaliação da disponibilidade de um determinado nutriente no solo, deve apresentar alto grau de relação com a planta, como rendimento, quantidade absorvida, entre outros, e ser rápido, de baixo custo, boa precisão e exatidão e com ampla capacidade extrativa (Corey, 1990).

A solução considerada padrão para extração desse elemento é o acetato de amônio $1 \mathrm{~mol} \mathrm{~L}^{-1}$ a pH 7,0 $\left(\mathrm{AcNH}_{4}\right)$, usado há mais de 50 anos (Metson, 1956). Além disso, o método é utilizado para extração simultânea de K, Ca e Mg, sendo considerado simples, rápido, de baixo custo e de boa exatidão (Haby et al., 1990). Contudo, para maior eficiência nos laboratórios de análises de solo, soluções que extraem diversos elementos simultaneamente têm sido preferidas, especialmente pela determinação dos elementos por técnicas de análise multielementar, como a espectrometria de emissão ótica por plasma acoplado indutivamente (ICP-OES).

Nos Estados do Rio Grande do Sul e Santa Catarina é utilizada a solução de Mehlich-1 $\left(\mathrm{M}_{1}\right)$ para extração de K no solo (Tedesco et al., 1995). Mehlich (1953) propôs o uso dessa solução para extração de $\mathrm{P}, \mathrm{K}, \mathrm{Ca}$, $\mathrm{Mg}, \mathrm{Na}, \mathrm{Mn}$ e Zn em solos ácidos. Os teores de K extraídos por essa solução correlacionaram-se com os extraídos pela solução de $\mathrm{AcNH}_{4}$, porém a solução de $\mathrm{M}_{1}$ extraiu teores menores. Para solos do Rio Grande do Sul, alguns estudos foram feitos para verificar a capacidade de extração da solução de Mehlich-1, relacionando com o rendimento de plantas (Kroth, 1998; Schlindwein \& Gianello, 2005; Bortolon et al., 2009a,b); no entanto, nesses trabalhos os solos não foram separados por classes de CTC para interpretação dos resultados, o que é feito para recomendação de adubação potássica para as culturas (SBCS/CQFS, 2004). Outro método que tem recebido atenção de pesquisadores é a solução de Mehlich-3 $\left(\mathrm{M}_{3}\right)$, por possibilitar a extração simultânea de $\mathrm{P}, \mathrm{K}, \mathrm{Ca}, \mathrm{Mg}$, $\mathrm{Na}, \mathrm{Mn}, \mathrm{Cu}$ e Zn (Mehlich, 1984), aumentando a eficiência dos laboratórios de análises de solo. No caso do K, alguns estudos foram feitos em solos do Rio Grande do Sul para verificar sua adequabilidade para uso em laboratórios de análises de solos, porém nesses estudos também não foi considerado o efeito da separação de classes de CTC na interpretação dos resultados (Kroth, 1998; Schlindwein \& Gianello, 2005; Bortolon et al., 2009a,b). Desse modo, há necessidade de verificar, além da capacidade extrativa dessas soluções, se estas são alteradas pela separação dos solos em classes de CTC numa ampla variabilidade de solos utilizados para produção agrícola no Estado do Rio Grande do Sul.

Outro método usado na determinação de K é a resina trocadora de íons (resina), o qual foi proposto 
por Amer at al. (1955) para extração simultânea de $\mathrm{P}, \mathrm{K}, \mathrm{Ca}$ e $\mathrm{Mg}$. O método da resina tem alto grau de correlação com o utilizado pelos laboratórios de análises de solos nos Estados do RS e SC (Kroth et al., 1998; Schlindwein et al., 2005; Bortolon et al., 2009b), além de ser amplamente utilizado pelos laboratórios de análises de solos do Estado de São Paulo há vários anos (Raij et al., 1986). Kroth (1998), estudando métodos de análise de solo para P, K, Ca e Mg em 20 solos do Rio Grande do Sul, observou que o método da resina extrai menos $\mathrm{K}$ em solos com altos teores, possivelmente devido à saturação da resina com essa alta quantidade de $\mathrm{K}$ no solo.

Os trabalhos publicados recentemente com solos do Rio Grande do Sul, na avaliação de métodos de análise de solo, para estimar a disponibilidade de K às plantas, não têm considerado o efeito da CTC na eficiência dos métodos (Kroth, 1998; Schlindwein \& Gianello, 2005; Bortolon et al., 2009a,b), sendo necessários estudos para verificar se a CTC altera a eficiência desses métodos na estimativa da disponibilidade de $\mathrm{K}$ às plantas, pois altera diretamente as recomendações de adubação potássica para as culturas. O objetivo deste trabalho foi avaliar a disponibilidade de K para o milho e para a soja em solos representativos do Estado do RS, por métodos com capacidade de extração multielementar, bem como verificar se a CTC altera a estimativa da disponibilidade de $\mathrm{K}$ no solo para as plantas.

\section{MATERIAL E MÉTODOS}

Foram utilizadas amostras de solos (camada de 0 $20 \mathrm{~cm}$ ) das classes mais representativas do Estado do RS (Quadro 1), com ampla variação de suas características físicas, químicas e mineralógicas, pertencentes ao banco de solos do Laboratório de Análises de Solos da UFRGS (Quadro 2). Foram utilizadas amostras de 21 solos no primeiro cultivo e de 14 solos no segundo cultivo, quando foram excluídas as amostras dos solos das unidades Vacaria I e II, Bom Jesus, São Jerônimo, São Gabriel I, Pelotas e Uruguaiana I, por apresentarem limitações ao crescimento e desenvolvimento das plantas de soja, devido à baixa disponibilidade de nutrientes após o cultivo do milho, principalmente $\mathrm{P}$.

Os solos foram passados em peneira com malha de $4 \mathrm{~mm}$ de diâmetro. Foram adicionados por $\mathrm{dm}^{3} \mathrm{de}$ solo: $0,1 \mathrm{mg}$ de $\mathrm{Mo}\left(\left(\mathrm{NH}_{4}\right)_{6} \mathrm{Mo}_{7} \mathrm{O}_{24} \cdot 4 \mathrm{H}_{2} \mathrm{O}\right), 0,5 \mathrm{mg}$ de $\mathrm{B}\left(\mathrm{H}_{3} \mathrm{BO}_{3}\right)$ e $5,0 \mathrm{mg}$ de $\mathrm{S}\left[\left(\mathrm{NH}_{4}\right)_{2} \mathrm{SO}_{4}\right]$, em solução. Não houve necessidade de correção da acidez nos solos em estudo, pois os teores de $\mathrm{Al}$ trocável estavam abaixo dos valores-limite para alterar o crescimento radicular. Além disso, não houve necessidade de adição de $\mathrm{P}$, pois os teores desse nutriente nos solos estavam enquadrados nas classes "alto" ou "muito alto" (SBCS/ CQFS, 2004).

Após a adição desses elementos, as amostras de solos foram novamente homogeneizadas. Foram, então, preparadas as unidades experimentais, constituídas de colunas de solo em tubos de PVC, de $30 \mathrm{~cm}$ de altura, com capacidade para $11 \mathrm{dm}^{3}$. Na parte inferior dos vasos foi colocado um suporte perfurado e uma tela de náilon com ocupação equivalente a $1 \mathrm{dm}^{3}$, para facilitar a drenagem. Um volume de $9 \mathrm{dm}^{3}$, de cada amostra de solo, foi transferido para o tubo de PVC. Os tratamentos (tipo de solo) foram dispostos em blocos casualizados, com três repetições. Durante 70 dias, os tubos de PVC foram mantidos ao tempo, cobertos com uma tela de náilon, para evitar o impacto direto das gotas da chuva na superfície do solo.

Quadro 1. Classificação, material de origem e locais de coleta das amostras de solos utilizadas no experimento

\begin{tabular}{|c|c|c|c|c|}
\hline Solo ${ }^{(1)}$ & Classificação brasileira ${ }^{(1)}$ & $\begin{array}{l}\text { Unidade de } \\
\text { mapeamento } \\
(2)\end{array}$ & $\begin{array}{r}\text { Material de } \\
\text { origem }^{(2)}\end{array}$ & $\begin{array}{c}\text { Município de } \\
\text { coleta }^{(3)}\end{array}$ \\
\hline VEo 1 & Vertissolo Ebânico órtico chernossólico & Aceguá & Siltito & Bagé \\
\hline SXe 2 & Planossolo Háplico eutrófico vértico & Bagé & Siltito & Bagé \\
\hline Cha 1 & Cambissolo Húmico alumínico típico & Bom Jesus & Eruptiva básica & Bom Jesus \\
\hline MTf & Chernossolo Argilúvio férrico típico & Ciríaco & Eruptiva básica & Dois Irmãos \\
\hline $\mathrm{LVd} 2$ & Latossolo Vermelho distrófico típico & Cruz Alta & Arenito & J. de Castilhos \\
\hline LVdf 1 & Latossolo Vermelho distroférrico típico & Durox & Eruptiva básica & André da Rocha \\
\hline LVaf & Latossolo Vermelho aluminoférrico típico & Erechim & Eruptiva básica & Erechim \\
\hline NVdf 1 & Nitossolo Vermelho distrófico latossólico & Estação & Eruptiva básica & Estrela \\
\hline PVAa 3 & Argissolo Vermelho-Amarelo alumínico típico & Júlio de Castilhos & Eruptiva básica & J. de Castilhos \\
\hline LVd 3 & Latossolo Vermelho distrófico típico & Passo Fundo & Eruptiva básica e arenito & Passo Fundo \\
\hline SGe 3 & Planossolo Hidromórfico eutrófico solódico & Pelotas & Sed. aluvial de granito & Camaquã \\
\hline LVdf 2 & Latossolo Vermelho distroférrico típico & Santo Ângelo & Eruptiva básica & Ijuí \\
\hline SXe 1 & Planossolo Háplico eutrófico típico & São Gabriel & Siltito & Pantano Grande \\
\hline PVd 7 & Argissolo Vermelho distrófico típico & São Jerônimo & Granito & Eldorado do Sul \\
\hline MEk & Chernossolo Ebânico carbonático vértico & Uruguaiana & Sedimento de basalto & Uruguaiana \\
\hline $\mathrm{LBa} 1$ & Latossolo Bruno alumínico câmbico & Vacaria & Eruptiva básica & Vacaria \\
\hline
\end{tabular}

(1) Embrapa (2006). ${ }^{(2)}$ Brasil (1973). ${ }^{(3)}$ Kroth (1998). 


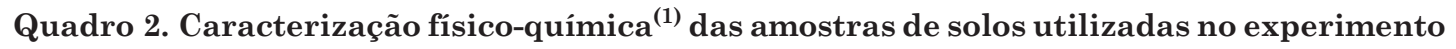

\begin{tabular}{|c|c|c|c|c|c|c|c|c|c|c|c|}
\hline $\begin{array}{l}\text { Unidade de } \\
\text { mapeamento }\end{array}$ & Argila & MO & $\mathrm{pH} \mathrm{H}_{2} \mathrm{O}$ & Índice SMP & $\mathbf{P}$ & $\mathbf{K}$ & $\mathrm{Zn}$ & $\mathbf{C u}$ & $\mathrm{Ca}^{2+}$ & $\mathrm{Mg}^{2+}$ & $\mathrm{Al}^{3+}$ \\
\hline & \multicolumn{2}{|c|}{$-\mathrm{g} \mathrm{dm} \mathrm{m}^{-3}-$} & & & \multicolumn{4}{|c|}{$-\mathrm{mg} \mathrm{dm} \mathrm{m}^{-3}$} & \multicolumn{2}{|c|}{$\mathrm{cmol}_{\mathrm{c}} \mathrm{dm}^{-3}$} & 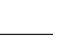 \\
\hline Santo Ângelo & 640 & 26 & 5,6 & 6,1 & 8,2 & 278 & 5,0 & 16,3 & 4,2 & 1,2 & 0,0 \\
\hline Durox & 530 & 38 & 6,1 & 6,4 & 7,9 & 301 & 5,9 & 7,0 & 8,2 & 2,4 & 0,0 \\
\hline Cruz Alta I & 240 & 16 & 5,5 & 6,3 & 13,7 & 76 & 4,0 & 3,2 & 2,7 & 0,8 & 0,0 \\
\hline Cruz Alta II & 250 & 17 & 5,7 & 6,4 & 36,6 & 81 & 2,3 & 2,3 & 3,4 & 0,9 & 0,0 \\
\hline Vacaria I & 540 & 58 & 5,9 & 6,1 & 6,5 & 375 & 6,9 & 12,1 & 9,5 & 3,0 & 0,0 \\
\hline Vacaria II & 500 & 48 & 5,9 & 6,1 & 11,4 & 545 & 6,7 & 10,7 & 8,7 & 2,7 & 0,0 \\
\hline Uruguaiana I & 240 & 31 & 5,5 & 6,2 & 8,4 & 91 & 5,2 & 4,5 & 9,5 & 3,8 & 0,0 \\
\hline Uruguaiana II & 250 & 31 & 5,5 & 6,2 & 14,4 & 107 & 4,5 & 5,1 & 11,0 & 3,8 & 0,0 \\
\hline Bom Jesus & 230 & 107 & 6,5 & 6,1 & 12,4 & 228 & 0,7 & 0,2 & 16,8 & 3,6 & 0,0 \\
\hline Bagé & 200 & 24 & 5,5 & 6,1 & 44,4 & 136 & 2,6 & 2,1 & 6,1 & 2,8 & 0,0 \\
\hline Aceguá & 320 & 46 & 5,7 & 6,2 & 28,2 & 355 & 5,6 & 2,4 & 11,8 & 4,4 & 0,0 \\
\hline São Gabriel I & 180 & 21 & 5,6 & 6,5 & 14,1 & 99 & 3,8 & 3,5 & 3,8 & 1,2 & 0,0 \\
\hline São Gabriel II & 160 & 20 & 5,3 & 6,2 & 25,9 & 34 & 4,0 & 4,0 & 3,7 & 0,9 & 0,4 \\
\hline São Jerônimo I & 250 & 21 & 5,6 & 6,5 & 15,5 & 125 & 4,2 & 3,3 & 2,9 & 0,9 & 0,0 \\
\hline São Jerônimo II & 240 & 20 & 5,4 & 6,4 & 27,3 & 103 & 3,6 & 3,1 & 2,6 & 0,8 & 0,4 \\
\hline Passo Fundo & 280 & 26 & 5,6 & 6,0 & 28,1 & 113 & 4,1 & 4,1 & 4,9 & 1,2 & 0,0 \\
\hline Pelotas & 100 & 19 & 5,3 & 6,2 & 29,1 & 62 & 4,5 & 3,3 & 2,6 & 0,6 & 0,4 \\
\hline Ciríaco & 400 & 37 & 5,8 & 6,0 & 12,8 & 316 & 7,8 & 5,2 & 14,3 & 4,9 & 0,0 \\
\hline Júlio de Castilhos & 510 & 28 & 5,5 & 6,0 & 18,3 & 248 & 6,0 & 4,0 & 5,2 & 1,5 & 0,0 \\
\hline Erechim & 560 & 38 & 5,7 & 6,0 & 15,3 & 174 & 6,0 & 7,0 & 7,7 & 1,8 & 0,0 \\
\hline Estação & 430 & 29 & 5,7 & 6,1 & 18,8 & 289 & 6,0 & 5,2 & 5,9 & 1,3 & 0,0 \\
\hline
\end{tabular}

(1) Tedesco et al. (1995) - MO: matéria orgânica (combustão úmida); P e K disponíveis extraídos pela solução de Mehlich-1; Cu e $\mathrm{Zn}$ disponíveis extraídos pela solução de $\mathrm{HCl} 0,1 \mathrm{~mol} \mathrm{~L}^{-1}$; $\mathrm{Ca}, \mathrm{Mg}$ e $\mathrm{Al}$ trocáveis extraídos pela solução de $\mathrm{KCl} 1,0$ mol L ${ }^{-1}$.

Antes da semeadura das culturas do milho e da soja, foram coletadas amostras de solo da camada superficial (aproximadamente de 0 a $20 \mathrm{~cm}$ de profundidade), de aproximadamente $0,25 \mathrm{dm}^{3}$, utilizando um trado calador. Após a coleta, a amostra de solo, contida em cada unidade experimental (na camada de $0-20 \mathrm{~cm}$ ), foi revolvida, com a finalidade de homogeneização, e compactada levemente (esse procedimento foi feito antes de cada cultivo). As amostras de solo foram secas em estufa com circulação forçada de ar, durante $72 \mathrm{~h}$, à temperatura de $45^{\circ} \mathrm{C}$. Posteriormente, o solo foi moído em gral de porcelana e tamisado em peneira com malha de $2 \mathrm{~mm}$ de diâmetro.

Nas colunas de solo, no primeiro cultivo, foi semeado milho (Zea mays), utilizando-se seis sementes por vaso. Após a emergência das plântulas, foi feito o desbaste, mantendo-se três plantas por vaso. A adubação nitrogenada foi feita com solução de ureia, aplicada na superfície do solo, na dose de $100 \mathrm{mg} \mathrm{dm}^{-3}$ de $\mathrm{N}$, parcelada em três aplicações, sendo $1 / 3$ na semeadura, $1 / 3$ aos 15 dias e $1 / 3$ aos 30 dias da semeadura. Após 45 dias de cultivo, as plantas foram cortadas pelo caule, a $1 \mathrm{~cm}$ da superfície do solo, e secas em estufa à temperatura de $65^{\circ} \mathrm{C}$, até peso constante. No segundo cultivo foi semeada soja (Glycine max), utilizando-se, por vaso, seis sementes inoculadas com rizóbio. Após a emergência das plantas, foi feito o desbaste, mantendo-se três plantas por vaso. Aos 45 dias de cultivo as plantas foram cortadas a $1 \mathrm{~cm}$ da superfície do solo e secas em estufa à temperatura de $65^{\circ} \mathrm{C}$ até peso constante. Em ambos os cultivos, não foi necessário fazer controle fitossanitário. Nos períodos de baixa precipitação pluvial, durante os cultivos, foi feita a irrigação com água deionizada. A água foi adicionada vagarosamente até o início do escorrimento pelo orifício de drenagem, localizado na parte inferior do vaso. A matéria seca da parte aérea de ambos os cultivos foi pesada e moída. A determinação dos teores dos nutrientes, na parte aérea das plantas de milho e soja, foi feita no extrato nítrico-perclórico, conforme descrito por Tedesco et al. (1995).

Nas amostras de solo coletadas antes dos cultivos, foi extraído o K disponível pelas soluções de $\mathrm{AcNH}_{4}$ (Metson, 1956), de $\mathrm{M}_{1}$ (Mehlich, 1953 modificado por Tedesco et al., 1995), de $\mathrm{M}_{3}$ (Mehlich, 1984) e por resina mista (Tedesco et al., 1995). A determinação do $\mathrm{K}$ foi feita por espectrofotometria de emissão atômica, utilizando espectrofotômetro de absorção atômica em modo de emissão.

Foram estabelecidas regressões lineares entre os teores extraídos de $\mathrm{K}$ pelos métodos em estudo e a quantidade acumulada de $\mathrm{K}$ pelas culturas do milho (primeiro cultivo) e da soja (segundo cultivo), considerando todos os solos e separando-os em classes de CTC a pH 7,0 ( $\left.\leq 5,0 ; 5,1-15,0 ;>15,1 \mathrm{cmol}_{\mathrm{c}} \mathrm{dm}^{-3}\right)$, estabelecidas para a recomendação de adubação potássica para as culturas cultivadas em solos dos Estados do Rio Grande do Sul e de Santa Catarina 
(SBCS/CQFS, 2004). A capacidade preditiva para avaliação de K disponível pelos diferentes métodos foi feita, principalmente, pela análise dos coeficientes de determinação, e a diferença entre os coeficientes foi avaliada pelo teste de Tukey a $5 \%$. A comparação da capacidade extrativa de $\mathrm{K}$ do solo foi feita por correlação, para verificar o grau de associação entre as variáveis; as diferenças entre os métodos foram avaliadas pelo teste t, tendo como padrão de comparação a solução de $\mathrm{AcNH}_{4}$ e a solução de $\mathrm{M}_{1}$. A definição dessas comparações se deve a duas razões: a solução de $\mathrm{AcNH}_{4}$ é considerada a solução-padrão de extração de K no solo (Haby et al., 1990); e a solução de $\mathrm{M}_{1}$ é o método atualmente empregado na avaliação da disponibilidade de $\mathrm{K}$ no solo para as plantas nos laboratórios de análises de solo dos Estados do RS e de SC.

\section{RESULTADOS E DISCUSSÃO}

\section{Comparação de métodos de extração}

Os teores extraídos de $\mathrm{K}$ variaram, no primeiro cultivo, de 52 a $504 \mathrm{mg} \mathrm{dm}^{-3}$ com a solução de $\mathrm{AcNH}_{4}$; de 34 a $545 \mathrm{mg} \mathrm{dm}^{-3}$ com a solução de $\mathrm{M}_{1}$; de 45 a $503 \mathrm{mg} \mathrm{dm}^{-3}$ com a solução de $\mathrm{M}_{3}$; e de 12 a $310 \mathrm{mg} \mathrm{dm}^{-3}$ com a resina. No segundo cultivo, os teores extraídos de $\mathrm{K}$ variaram de 31 a $314 \mathrm{mg} \mathrm{dm}^{-3}$ com a solução de $\mathrm{AcNH}_{4}$; de 31 a $243 \mathrm{mg} \mathrm{dm}^{-3}$ com a solução de $\mathrm{M}_{1}$; de 29 a $283 \mathrm{mg} \mathrm{dm}{ }^{-3}$ com a solução de $\mathrm{M}_{3}$; e de 5 a $139 \mathrm{mg} \mathrm{dm}^{-3}$ com a resina (Quadro 3).

A relação entre os teores de $\mathrm{K}$ extraídos pelas soluções de $\mathrm{AcNH}_{4}$ e de $\mathrm{M}_{1}$ é mostrada na figura 1a.
Os coeficientes de correlação entre os teores extraídos pelas soluções foram de 0,92 e 0,97 para os solos coletados antes do primeiro e segundo cultivos, respectivamente. A capacidade extratora da solução de $\mathrm{M}_{1}$ foi menor que a da solução de $\mathrm{AcNH}_{4}$. Em média, os teores de K extraídos foram $19 \%$ menores no primeiro cultivo e $24 \%$ menores no segundo (Quadro 3). Esses resultados concordam, em geral, com os da literatura (Wang et al., 2004; Zbíral \& Nemec, 2005; Hosseinpur \& Samavati, 2008). O tempo de agitação das amostras (15 min para a solução de $\mathrm{AcNH}_{4}$ e 5 min para a solução de $\mathrm{M}_{1}$ ) também pode ser outro fator a influenciar na maior extração pela solução de $\mathrm{AcNH}_{4}$, por possibilitar maior tempo de contato e de troca iônica entre o $\mathrm{K}$ contido nos sítios de troca e o $\mathrm{NH}_{4}$ (Habby at al., 1990).

A relação entre os teores extraídos pela solução de $\mathrm{AcNH}_{4}$ e pela solução de $\mathrm{M}_{3}$ é mostrada na figura $1 \mathrm{~b}$. Os coeficientes de correlação foram de 0,98 e de 0,99, respectivamente, para o primeiro e segundo cultivos. Os teores de $\mathrm{K}$ extraídos pela solução de $\mathrm{M}_{3}$, em média, não diferiram estatisticamente dos extraídos pela solução de $\mathrm{AcNH}_{4}$. Provavelmente, o maior teor extraído em relação ao $\mathrm{M}_{1}$ deve-se à composição e ao maior $\mathrm{pH}(\mathrm{pH} 2,5)$ da solução de $\mathrm{M}_{3}$ e por esta solução conter acetato de amônio, formado a partir da adição de todos os reagentes que compõem o método, aumentando, desse modo, os teores extraídos de K (Mehlich, 1984). Os resultados obtidos neste trabalho são concordantes com os obtidos na literatura (Evans \& McGuire, 1990; Alva, 1993; Wang et al., 2004; Zbíral \& Nemec, 2005; Hosseinpur \& Samavati, 2008).

A relação entre os teores extraídos de K pela solução de $\mathrm{AcNH}_{4}$ e pela resina é mostrada na figura 1c. Os

Quadro 3. Estatística descritiva dos teores de potássio extraído do solo por diferentes métodos antes do primeiro (milho) e segundo (soja) cultivos, teor e acúmulo de potássio na parte aérea e produção de matéria seca do milho e da soja

\begin{tabular}{|c|c|c|c|c|c|c|c|}
\hline \multirow{2}{*}{ Parâmetro } & \multicolumn{4}{|c|}{ Potássio no solo } & \multicolumn{2}{|c|}{ Potássio na planta } & \multirow{2}{*}{$\begin{array}{c}\text { Matéria } \\
\text { seca }\end{array}$} \\
\hline & $\mathrm{AcNH}_{4}$ & Mehlich-1 & Mehlich-3 & Resina & Tecido & Acumulado & \\
\hline & 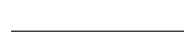 & $\mathrm{mg}$ & $\mathrm{dm}^{-3}-$ & 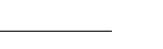 & $\%$ & $\mathrm{mg} / \mathrm{vaso}$ & g/vaso \\
\hline & \multicolumn{6}{|c|}{ ing um } & \\
\hline Média & $243(100)^{(1)}$ & $197(81)$ & $236(97)$ & $108(44)$ & 2,1 & 291,4 & 14,0 \\
\hline Mínimo & 52 & 34 & 45 & 12 & 0,5 & 54,0 & 3,6 \\
\hline Máximo & 504 & 545 & 503 & 310 & 3,7 & 630,3 & 21,1 \\
\hline \multirow[t]{2}{*}{ Mediana } & 165 & 136 & 186 & 77 & 2,4 & 252,2 & 14,3 \\
\hline & \multicolumn{7}{|c|}{ Soja } \\
\hline Média & $135(100)$ & $102(76)$ & $127(94)$ & $50(37)$ & 1,6 & 232,6 & 13,2 \\
\hline Mínimo & 31 & 31 & 29 & 5 & 0,6 & 57,3 & 9,0 \\
\hline Máximo & 314 & 243 & 283 & 139 & 3,2 & 576,0 & 18,0 \\
\hline \multirow[t]{2}{*}{ Mediana } & 130 & 93 & 124 & 34 & 1,6 & 206,2 & 13,8 \\
\hline & \multicolumn{7}{|c|}{ Média dos dois cultivos } \\
\hline Média & $204(100)$ & $166(91)$ & $197(97)$ & $89(43)$ & 2,0 & 271,9 & 13,4 \\
\hline Mínimo & 31 & 31 & 29 & 5 & 0,5 & 54,0 & 3,6 \\
\hline Máximo & 504 & 545 & 503 & 310 & 3,7 & 630,3 & 21,1 \\
\hline Mediana & 164 & 125 & 165 & 69 & 2,1 & 252,2 & 14,3 \\
\hline
\end{tabular}

(1) Valores entre parênteses na linha indicam a percentagem em relação à solução de acetato de amônio. 
coeficientes de correlação obtidos entre os métodos foram de 0,90 e de 0,85 , respectivamente, para o primeiro e segundo cultivos. Os teores extraídos pela resina foram aproximadamente $50 \%$ menores (Quadro 3). Os resultados deste trabalho destoam dos obtidos por Raij et al. (1986), os quais não encontraram diferença entre a resina e a solução de $\mathrm{AcNH}_{4}$. No entanto, esses autores utilizaram resinas em esferas e, por conseguinte, a área superficial específica entre esferas e membranas é diferente, gerando resultados destoantes (Kroth, 1998). Além disso, a variabilidade mineralógica dos solos utilizados neste trabalho é maior do que a daqueles utilizados por Raij et al. (1986), alterando, desse modo, os teores extraídos.

Na figura $1 \mathrm{~d}$ é mostrada a relação entre os teores de $\mathrm{K}$ extraídas pela soluções de $\mathrm{M}_{1}$ e de $\mathrm{M}_{3}$. Os coeficientes de correlação entre as soluções foram de 0,93 e de 0,96 , respectivamente para amostras coletadas antes do primeiro e segundo cultivos. Resultados semelhantes foram obtidos por Kroth (1998) em 60 amostras de 20 solos do RS; Evans \& McGuire (1990), em 50 solos do Alabama (Alva, 1993; Wang et al., 2004). Os maiores teores extraídos pela solução de $\mathrm{M}_{3}$ (Quadro 3) devem-se à formação de $\mathrm{AcNH}_{4}$ durante a composição da solução, resultando em maiores teores de K extraídos (Mehlich, 1984). Contudo, quando foram comparados os teores médios extraídos agrupando-se os dois cultivos, as soluções não diferiram entre si (Quadro 3). Isso possivelmente está associado à inclusão de maior número de solos na relação, fazendo com que aumente o coeficiente de correlação, reduzindo a diferença entre os teores médios extraídos de K (Kroth, 1998).

A comparação da capacidade extratora da solução de $\mathrm{M}_{1}$ e da resina é mostrada na figura 1e. Os coeficientes de correlação entre os dois métodos foram de 0,99 e 0,96 , respectivamente, em amostras de solo coletadas antes do primeiro e segundo cultivos. Em média, os teores de $\mathrm{K}$ extraídos pela resina foram aproximadamente $50 \%$ menores do que os obtidos com a solução de $\mathrm{M}_{1}$ (Quadro 3). Considerando o princípio do método da resina e o tempo de agitação das amostras, os teores deveriam ser semelhantes. A área superficial específica da resina trocadora de cátions pode ter sido subestimada. Contudo, se fosse aceita essa justificativa, a regressão apresentaria tendência curvilinear, pois, para teores baixos de $\mathrm{K}$, os teores seriam semelhantes aos extraídos pela solução de $\mathrm{M}_{1}$, e para teores elevados, menores que os da solução de $\mathrm{M}_{1}$. A figura 1c, no entanto, mostra que a relação foi linear e a dispersão dos pontos foi baixa, sobretudo em amostras coletadas antes do primeiro cultivo, em que os teores de $\mathrm{K}$ foram maiores do que naquelas antes do segundo cultivo. Os menores teores de K extraídos pelo método da resina podem estar associados ao mecanismo de extração do método. As soluções
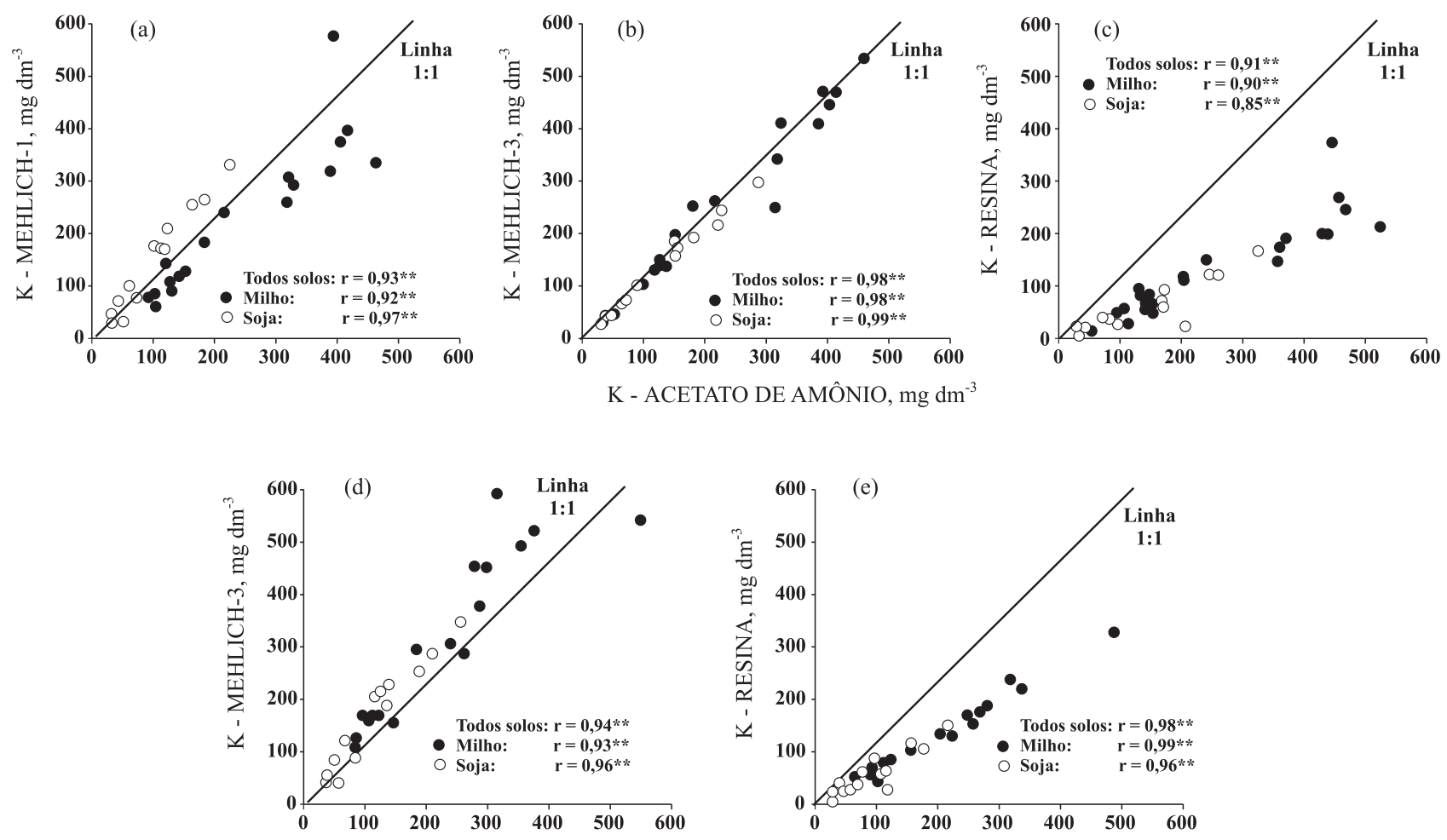

$\mathrm{K}$ - MEHLICH-1, $\mathrm{mg} \mathrm{dm}^{-3}$

Figura 1. Correlação entre os teores de potássio extraídos pelos métodos em amostras de solos coletadas antes do primeiro, com milho, e do segundo cultivo, com soja. 
ácidas $\left(\mathrm{M}_{1}\right.$ e $\left.\mathrm{M}_{3}\right)$ e neutra $\left(\mathrm{AcNH}_{4}\right)$ deslocam a maior parte do $\mathrm{K}$ trocável, considerado disponível pelos estudos de correlação, enquanto na extração com resina ocorre a remoção por troca dos íons $\mathrm{K}$ na solução e que podem passar à solução (Tedesco et al., 1995). Essa diferença nos mecanismos de extração proporciona diferentes teores extraídos entre os métodos. O mesmo foi observado por outros estudos conduzidos com solos do Estado do RS (Kroth, 1998; Schlindwein \& Gianello, 2005; Bortolon et al., 2009a,b).

Em geral, os métodos tiveram alto grau de relação quando comparados dois a dois; no entanto, devido à menor amplitude de extração e de nutrientes possíveis de serem extraídos simultaneamente, o método da resina apresenta certa desvantagem em relação aos outros, por reduzir a eficiência dos laboratórios de análises de solo. Além disso, o custo relativamente elevado para aquisição das membranas é um fator a ser considerado na implementação de um método de análise de solo. A menor amplitude de extração do método da resina ocasiona faixas de fertilidade menores, o que propicia o aumento no erro analítico, se comparado a um método com maior capacidade extrativa.

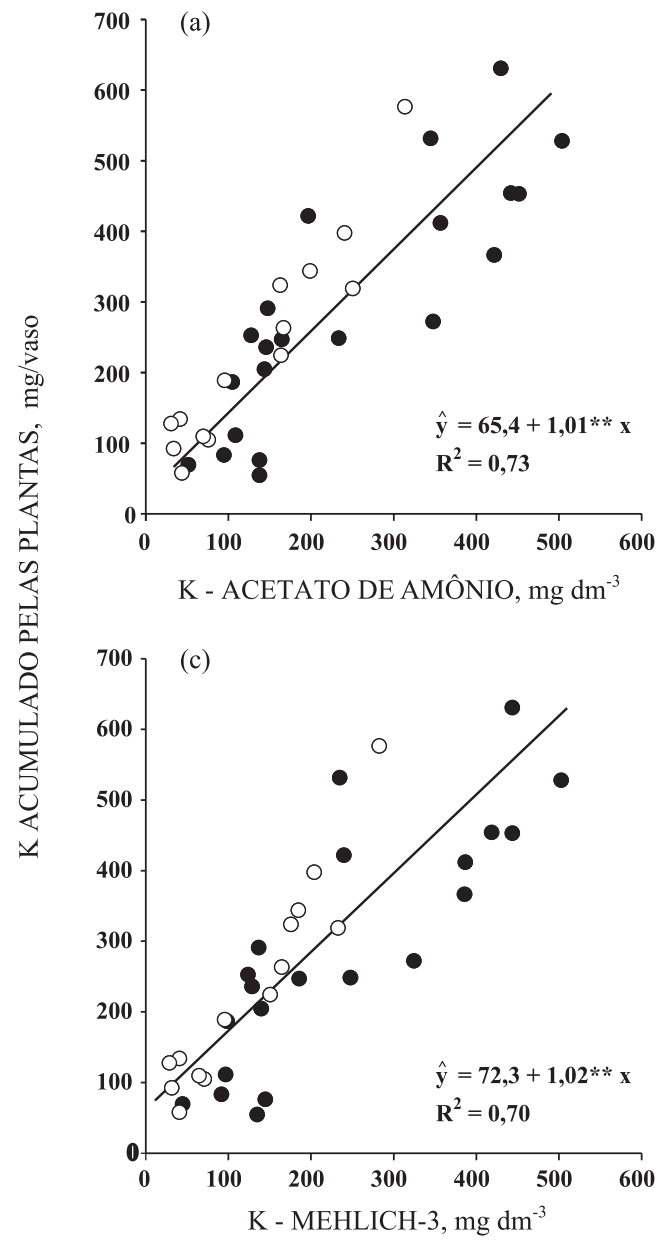

\section{Relação entre os teores extraídos pelas soluções e a absorção pelas plantas}

A relação entre o teor de $\mathrm{K}$ no solo extraído pela solução de $\mathrm{AcNH}_{4}$ e o $\mathrm{K}$ acumulado pelas plantas é mostrada na figura $2 \mathrm{a}$. Os coeficientes de determinação foram de 0,72 e de 0,90, para o milho e a soja, respectivamente (Quadro 4). A relação entre os teores de $\mathrm{K}$ extraídos pela solução de $\mathrm{M}_{1}$ e o $\mathrm{K}$ acumulado no primeiro e segundo cultivos é mostrada na figura $2 \mathrm{~b}$. Os coeficientes de determinação foram de 0,75 e 0,87, para o milho e a soja, respectivamente. A relação entre os teores de $\mathrm{K}$ extraídos pela solução de $\mathrm{M}_{3}$ e o K acumulado no primeiro e segundo cultivos é mostrada na figura 2c. Os coeficientes de determinação foram de 0,70 e 0,90, para o milho e a soja, respectivamente. A relação entre os teores de $\mathrm{K}$ extraídos pela resina e o K acumulado pelas plantas no primeiro e segundo cultivos é mostrada na figura $2 \mathrm{~d}$. Os coeficientes de determinação foram de 0,72 e 0,71 respectivamente para o milho e a soja. Todos os métodos foram eficientes na estimativa da disponibilidade de $\mathrm{K}$ às plantas em solos do RS, exceto o método da resina na cultura da soja. Resultados semelhantes foram obtidos por Kroth (1998), Schlindwein \& Gianello (2005) e Bortolon et al. (2009b) para solos do RS.

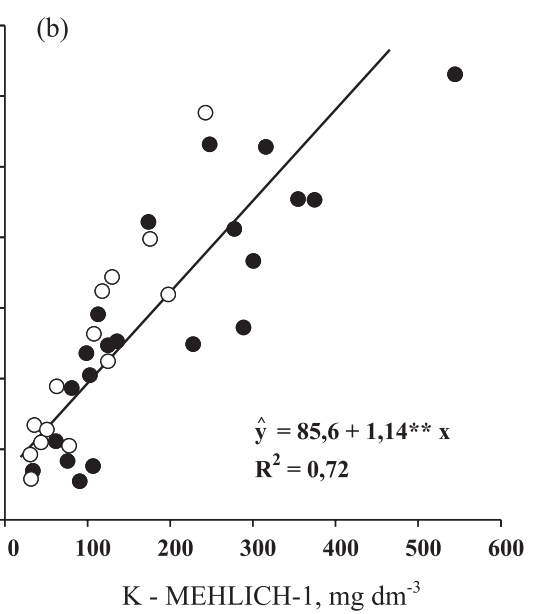

(d)

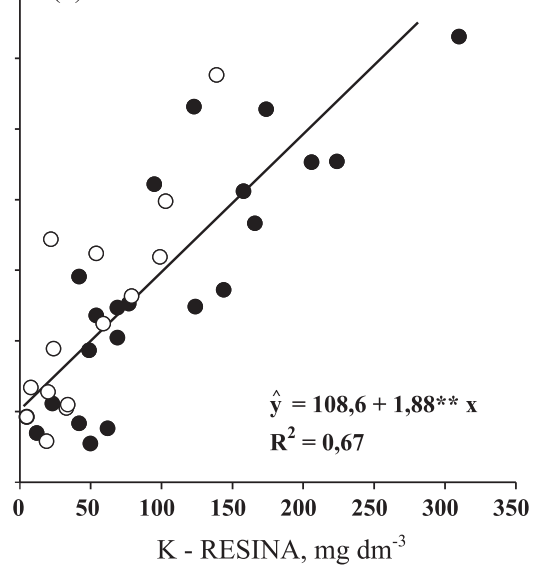

Figura 2. Relação entre os teores de potássio extraídos pelas soluções de acetato de amônio (a), Mehlich-1 (b), Mehlich-3 (c) e por resina (d) e a quantidade acumulada de potássio pelas plantas de milho e soja. 
A separação dos solos por classes de CTC estabelecidas no Manual de adubação e de calagem para os Estados do Rio Grande do Sul e de Santa Catarina (SBCS/CQFS, 2004) aumentou os valores dos coeficientes de determinação na estimativa do $\mathrm{K}$ disponível às plantas, sendo maiores nos solos com CTC > 15, $1 \mathrm{cmol}_{\mathrm{c}} \mathrm{dm}^{-3}$ (Quadro 4). Entretanto, o maior valor observado pode estar associado ao menor número de solos nessa classe em relação às demais, aumentando, consequentemente, o coeficiente de determinação pela menor variabilidade dos solos. Além disso, essa diferença foi observada somente no primeiro cultivo. No segundo, não houve diferença para a separação de classes, exceto para o método da resina. Em geral, dentro de cada classe de CTC e considerando todos os solos, os métodos não diferiram entre si na capacidade de avaliar a disponibilidade de $\mathrm{K}$ às plantas (Quadro 4) .

Quadro 4. Coeficiente de determinação $\left(R^{2}\right)$ de ajuste linear obtido entre os teores de potássio extraídos pelos métodos e o potássio acumulado pelas plantas em diferentes classes de capacidade de troca de cátions (CTC)

\begin{tabular}{|c|c|c|c|}
\hline \multirow{2}{*}{ Relações } & \multirow{2}{*}{ Todos os solos } & \multicolumn{2}{|c|}{$\mathrm{CTC}_{\mathrm{pH} 7,0}\left(\mathrm{cmol}_{\mathrm{c}} \mathrm{dm}^{-3}\right)$} \\
\hline & & $5,1-15,0$ & $>15,1$ \\
\hline $\mathrm{K}$ acumulado & & Milho $\left(\mathrm{R}^{2}\right)$ & \\
\hline $\mathrm{NH}_{4} \mathrm{Ac}$ & $0,72^{* *}$ aB & $0,60^{* *} \mathrm{aC}$ & $0,89 * *$ aA \\
\hline Mehlich-1 & $0,75^{* *} \mathrm{aB}$ & $0,61 * * \mathrm{aC}$ & $0,91^{* *}$ aA \\
\hline Mehlich-3 & $0,70^{* *} \mathrm{aB}$ & $0,53^{* *} \mathrm{aC}$ & $0,92^{* *}$ aA \\
\hline Resina & $0,72^{* *} \mathrm{aB}$ & $0,58^{* *} \mathrm{aC}$ & $0,90 * *$ aA \\
\hline $\mathrm{K}$ acumulado & & Soja $\left(R^{2}\right)$ & \\
\hline $\mathrm{NH}_{4} \mathrm{Ac}$ & $0,90^{* *}$ aA & $0,95 * *$ aA & $0,99 * *$ aA \\
\hline Mehlich-1 & $0,87^{* *}$ aA & $0,93^{* *}$ aA & $0,87^{* *}$ aA \\
\hline Mehlich-3 & $0,90^{* *} \mathrm{aA}$ & $0,96^{* *} \mathrm{aA}$ & $0,99^{* *}$ aA \\
\hline Resina & $0,71^{* *} \mathrm{bB}$ & $0,74^{* *} \mathrm{aB}$ & $0,99 * *$ aA \\
\hline
\end{tabular}

**: significativo $(p<0,01)$; valores seguidos por letras minúsculas na coluna e maiúsculas na linha diferem entre si pelo teste de Tukey a $5 \%$.

Todos os métodos de extração de K estudados foram altamente correlacionados entre si e apresentaram baixa dispersão de pontos quando comparados dois a dois, bem como estimaram a disponibilidade de K às plantas satisfatoriamente, considerando a grande variabilidade mineralógica dos solos utilizados neste estudo. Um método adequado para avaliar a disponibilidade de nutrientes às plantas deve ser eficiente para uma grande variabilidade de tipos de solo e diferentes tipos de cultura.

\section{CONCLUSÕES}

1. A disponibilidade de potássio para as plantas em solos do Rio Grande do Sul pode ser feita pelos métodos testados no presente trabalho.
2. Não houve diferença entre os métodos na predição da disponibilidade de K quando os solos foram separados em classes de capacidade de troca de cátions.

3. O método da resina, embora não diferindo dos demais na avaliação da disponibilidade de Kàs plantas, apresenta desvantagem em relação às soluções de acetato de amônio, Mehlich-1 e Mehlich-3, pela menor capacidade extrativa, o que resulta em classes de disponibilidade menores e propicia maior erro analítico.

\section{LITERATURA CITADA}

ALVA, A.K. Comparisons of Mehlich 3, Mehlich 1, ammonium bicarbonate-DTPA, $1.0 \mathrm{M}$ ammonium acetate, and $0.2 \mathrm{M}$ ammonium chloride for extraction of calcium, magnesium, phosphorus, and potassium for a wide range soils. Comm. Soil Sci. Plant Anal., 24:603-612, 1993.

AMER, T.; BOULDING, D.R.; BLACK, C.A. \& DUKE, F.R. Characterization of soil phosphorus by anion exchange resin adsorption and P32 equilibration. Plant Soil, 6:391408, 1955 .

BORTOLON, L.; GIANELLO, C. \& SCHLINDWEIN, J.A. Avaliação da disponibilidade de fósforo no solo para o milho pelos métodos Mehlich-1 e Mehlich-3. Sci. Agr., 10:305-312, 2009a.

BORTOLON, L.; SCHLINDWEIN, J.A. \& GIANELLO, C. Métodos de extração de fósforo e potássio no solo sob sistema plantio direto. Ci. Rural, 39:2400-2407, 2009b.

COREY, R.B. Physical-chemical aspects of nutrient availability. In: WESTWERMAN, R.L., ed. Soil testing and plant analysis. Madison, Wisconsin, SSSA, 1990. p.11-27.

EVANS, C.E. \& McGUIRE, J.A. Comparison of soil test extractants on Alabama soils. Comm. Soil Sci. Plant Anal., 21:1037-1050, 1990.

HABY, V.A.; RUSSELLE, M.P. \& SKOGLEY, E.O. Testing soils for potassium, calcium and magnesium. In: WESTWERMAN, R.L., ed. Soil testing and plant analysis. Madison, Wisconsin, SSSA, 1990. p.181-227.

HOSSEINPUR, A.R. \& SAMAVATI, M. Evaluation of chemical extractans for the determination of available potassium. Comm. Soil Sci. Plant Anal., 39:1559-1570, 2008.

KROTH, P.L. Disponibilidade de fósforo no solo para as plantas e fatores que afetam a extração por resina de troca em membrana. Porto Alegre, Universidade Federal do Rio Grande do Sul, 1998.168p. (Tese de Mestrado)

MEHLICH, A. Determination of $\mathrm{P}, \mathrm{Ca}, \mathrm{Mg}, \mathrm{K}, \mathrm{Na}$ and $\mathrm{NH} 4$ by North Carolina soil testing laboratories. Raleigh, University of North Carolina, 1953. 4p.

MEHLICH, A. Mehlich 3 soil test extractant: A modification of Mehlich 2 extractant. Comm. Soil Sci. Plant Anal., 15:1409$1416,1984$.

METSON, A.J. Methods of chemical analysis for survey samples. Wellington, D.S.I.R., 1956. 207p. (Soil Bureau Bulletin, 12) 
RAIJ. B.van; QUAGGIO, J.A. \& SILVA, N.M. Extraction of phosphorus, potassium, calcium, and magnesium from soils by an ion-exchange resin procedure. Comm. Soil Sci. Plant Anal., 17:547-566, 1986.

SCHLINDWEIN, J.A. \& GIANELLO, C. Doses de máxima eficiência econômica de fósforo e potássio para as culturas cultivadas no sistema plantio direto. R. Plantio Direto, 85:20-25, 2005 .

SOCIEDADE BRASILEIRA DE CIÊNCIA DO SOLO/ COMISSÃO DE QUÍMICA E FERTILIDADE DO SOLO . SBCS/CQFS. Manual de adubação e de calagem para os Estados do Rio Grande do Sul e de Santa Catarina. 10.ed. Porto Alegre, 2004. 400p.
TEDESCO, M. J.; GIANELLO, C.; BISSANI, C.A.; BOHNEN, H. \& VOLKWEISS, S.J. Análise de solo, plantas e outros materiais. 2.ed. Porto Alegre, Departamento de Solos da Universidade Federal do Rio Grande do Sul. 1995. 174p. (Boletim Técnico de Solos, 5)

WANG, J.J.; HARRELL, D.L.; HENDERSON, R.E. \& BELL, P.F. Comparison of soil-test extractants for phosphorus, potassium, calcium, magnesium, sodium, zinc, copper, manganese, and iron in Louisiana soils. Comm. Soil Sci. Plant Anal., 35:145-160, 2004.

ZBÍRAL, J. \& NEMEC, C. Comparison of Mehlich 2, Mehlich 3, CAL, Schachtschabel, $0.01 \mathrm{M} \mathrm{CaCl}_{2}$ and aqua regia extractants for determination of potassium in soils. Comm. Soil Sci. Plant Anal., 36:795-803, 2005. 